\title{
EFFECTS OF VISCOSITY AND ATP CONCENTRATION ON THE MOVEMENT OF REACTIVATED SEA-URCHIN SPERM FLAGELLA
}

\author{
By C. J. BROKAW \\ Division of Biology, California Institute of Technology, \\ Pasadena, California 91125
}

(Received I 8 November 1974)

\begin{abstract}
SUMMARY
I. Spermatozoa from the sea urchin, Lytechinus pictus, can be demembranated with solutions containing Triton X-100 and $5 \mathrm{~mm}-\mathrm{CaCl}_{2}$ and reactivated in ATP solutions containing low concentrations $\left(\mathrm{rO}^{-9} \mathrm{M}\right)$ of free $\mathrm{Ca}^{2+}$ ion to give symmetrical bending wave movements, even at very low ATP concentrations. At ATP concentrations of $0.01-0.02 \mathrm{mM}$ the reactivated spermatozoa have beat frequencies near $\mathrm{I} \mathrm{Hz}$, nearly normal bend angles, and wave-lengths about $50 \%$ longer than normal.

2. The effects of increased viscosity, obtained by addition of methyl cellulose to the reactivation solutions, on bend angle and beat frequency decrease with decreasing ATP concentration, and become almost undetectable at $0.01 \mathrm{mM} \mathrm{ATP.} \mathrm{On} \mathrm{the} \mathrm{other} \mathrm{hand,} \mathrm{the} \mathrm{effect} \mathrm{of} \mathrm{increased} \mathrm{viscosity} \mathrm{on}$ wavelength shows relatively little change with ATP concentration, although it is noticeably reduced at $0.01 \mathrm{mM} \mathrm{ATP}$.

3. These observations suggest that the beat frequency at low ATP concentrations is determined by an intrinsic rate-limiting process, in contrast to the viscosity-limited behaviour at high beat frequencies. Quantitative agreement with the experimental results is obtained with a model in which ATP concentration and viscosity each determine the rates of one step in a two-step reaction cycle which determines the beat frequency.

4. Two other models which can qualitatively explain the effects of ATP concentration and viscosity on flagellar beat frequency fail to show quantitative agreement with the experimental results. In one of these models, ATP concentration determines the maximum rate of shear between filaments. In the other, ATP concentration determines a time delay which is required to bring the active moment into phase with the elastic moments which would be expected to dominate the bending resistance at low beat frequencies.
\end{abstract}

\section{INTRODUCTION}

Effects of viscosity and adenosine-triphosphate (ATP) concentration on the form and frequency of the bending waves generated by ATP-reactivated sea-urchin sperm flagella were examined in earlier studies using glycerol-demembranated spermatozoa (Brokaw, 1966, 1967; Brokaw \& Josslin, 1973). Two recent improvements in techniques for ATP-reactivation of sperm Hagella now make it possible to obtain much 
better data from such experiments. Gibbons \& Gibbons (1972) found that a more complete removal of the flagellar membranes could be obtained using the detergent Triton X-100 instead of glycerol, giving a much higher percentage - close to $100 \%$ of successfully reactivated, motile spermatozoa. The symmetry of the bending waves of reactivated sperm flagella from some sea-urchin species has now been found to be sensitive to the concentrations of calcium ions in both the extraction and reactivation solutions (Brokaw, Josslin \& Bobrow, 1974; Brokaw \& Gibbons, 1975). Careful control of calcium ion concentrations makes it possible to obtain preparations in which most of the sperm flagella generate symmetrical bending waves and give regular wave movement even when reactivated at low ATP concentrations. Studies using these preparations provide new information relevant to the control of bending wave parameters by sea-urchin sperm flagella. Some of these studies have been briefly described elsewhere (Brokaw, 1974).

\section{MATERIALS AND METHODS}

Sea urchins (Lytechinus pictus) were collected in Mission Bay, California, during June and July. They were maintained on a diet of kelp (Macrocystis) in aquaria with flowing, filtered sea water at $10-15{ }^{\circ} \mathrm{C}$ for up to I year. Gamete shedding was induced by injection of $0.6 \mathrm{M}-\mathrm{KCl}$. The aboral surface of the urchin was rinsed with 0.5 $\mathrm{M}-\mathrm{NaCl}$, and spermatozoa were collected with a pipette as they emerged from the gonopores. The 'dry' spermatozoa were diluted with $\mathrm{I}-2$ volumes of cold $0.5 \mathrm{M}-\mathrm{NaCl}$ to form a suspension which could be sampled reproducibly. This suspension was stored on ice during a series of experiments. The quality of the reactivated movements obtained with a particular sperm sample usually deteriorated noticeably after about I h. This factor limited the amount of quantitative data which could be obtained from a particular sperm sample and was consequently a significant limitation on the precision of the results. Attempts to find more satisfactory conditions for sperm storage were unsuccessful.

Membrane removal was accomplished with an 'extraction solution' containing $0.15 \mathrm{M}-\mathrm{KCl}, 2 \mathrm{~mm}-\mathrm{MgSO}_{\text {, }} 2 \mathrm{~mm}$ dithiothreitol (DTT), $2 \mathrm{~mm}$ Tris buffer, $0.5 \mathrm{~mm}$

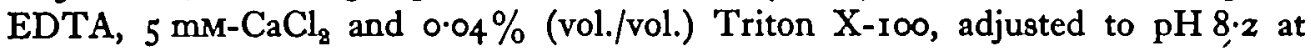
$16^{\circ} \mathrm{C}$. Sperm motility was observed in a 'reactivation solution' which normally contained $0.15 \mathrm{M}-\mathrm{KCl}, 2 \mathrm{~mm}-\mathrm{MgSO}_{4}, 2 \%$ (wt/vol.) polyethylene glycol (carbowax), $2 \mathrm{mM}$ DTT, $20 \mathrm{~mm}$ Tris buffer, $2.0 \mathrm{~mm}$ EGTA, $0.5 \mathrm{~mm}-\mathrm{CaCl}_{2}$ and variable amounts of ATP. The $\mathrm{pH}$ of the reactivation solution was also adjusted to $\mathrm{pH} 8.2$ at $16^{\circ} \mathrm{C}$. The reactivation solution was designed to have a low concentration of free calcium ions, approximately $1 \mathrm{O}^{-9} \mathrm{M}$. At $\mathrm{pH} 8 \cdot 2$ a significant amount of magnesium ion binding by EGTA will occur. Using stability constants from Sillen \& Martell (1964), the calculated concentration of free $\mathrm{Mg}^{2+}$ ion in a reactivation solution containing no ATP will be ${ }^{\prime} \cdot I$ mM. Addition of ATP will decrease the amount of free $\mathrm{Mg}^{2+}$ ion, so that the MgATP2- concentration will not increase in proportion to the added ATP unless additional magnesium is added to the solution. This effect is insignificant at ATP concentrations of $0.2 \mathrm{mM}$ or less, and has been neglected in most of the experiments. For the beat frequency measurements at higher ATP concentrations (Fig. I), ATP and $\mathrm{MgSO}_{4}$ were added to the reactivation solutions in the proportion of $\mathrm{I} I \mathrm{I}: \mathrm{I}$. 
With a free $\mathrm{Mg}^{2+}$ concentration of $\mathrm{I} \cdot \mathrm{I} \mathrm{mM}$ and a $\mathrm{MgATP}^{2-}$ stability constant of $\mathrm{I} \cdot 0 \times \mathrm{IO}^{4} \mathrm{M}^{-1}$ (Sillen \& Martell, I964) this procedure will result in a $\mathrm{MgATP}^{2-}$ concentration equal to the amount of additional $\mathrm{MgSO}_{4}$ added with the ATP. Some control experiments in which beat frequencies of sperm flagella were measured at lower $\mathrm{MgSO}_{4}$ concentrations were carried out to test these calculations. The results were consistent with the assumption that beat frequency is determined by MgATP2concentration and a stability constant for $\mathrm{MgATP}^{2-}$ of $\mathrm{I}_{4}^{\cdot} \times 1 \mathrm{10}^{4} \mathrm{M}^{-1}$, which is not a significant difference. However, if the stability constant is considerably larger, as suggested by some measurements $\left(3.4 \times 10^{4} \mathrm{M}^{-1}\right.$ at $17{ }^{\circ} \mathrm{C}$; Sari, Ragot \& Belaich, 1973; see also references in Godt, 1974), the calculated MgATP ${ }^{2-}$ concentrations in the reactivation solutions at high ATP concentrations will be too low, but not by more than $10 \%$.

Reactivation solutions of increased viscosity were obtained by addition of methyl cellulose of high molecular weight (Fisher M-28I, $4000 \mathrm{cP}$ ) to the solutions. Viscosities were measured with capillary viscometers at $16{ }^{\circ} \mathrm{C}$ (Brokaw, I966), but no shear-rate correction was used as the present results were limited to relatively low frequencies and viscosities.

All preparations and observations were carried out in a room maintained at $16^{\circ} \mathrm{C}$. A $5 \mu 1$ sample of spermatozoa was added to $0.25 \mathrm{ml}$ of extraction solution and agitated gently to suspend the spermatozoa. After $30 \mathrm{sec}$ incubation in the extraction solution a 5-10 $\mu$ l portion of this suspension was mixed with $\mathrm{I} \cdot 0 \mathrm{ml}$ of reactivation solution, and a drop of this suspension was transferred to a slide on the microscope stage. The extraction and reactivation procedure was repeated to prepare each sample observed under the microscope.

Beat frequency measurements were made by observing spermatozoa swimming at the upper surface of an open drop of solution, using a $\times 16$ objective and dark-field illumination. Beat frequencies below about $2 \mathrm{~Hz}$ were measured by timing ro beats with a recording electronic timer. Beat frequencies above $2 \mathrm{~Hz}$ were measured stroboscopically, as in previous work. In some experiments, bend angles were estimated visually by comparing the waveforms of spermatozoa observed with stroboscopic illumination with a chart showing waveforms drawn with bend angles of $x \cdot 0$ I. $5,2 \cdot 0$ radians, etc. The feasibility and accuracy of this procedure depends on the availability of preparations in which the spermatozoa are generating symmetrical bending waves.

Photographs were obtained, for measurements of wavelengths and bend angles, using a $\times 4^{\circ}$ oil-immersion objective, with a coverglass over the drop of sperm suspension. Although most of the spermatozoa stick to either the slide or cover glass under these conditions, a sufficient number of freely swimming spermatozoa could usually be found for photography by careful searching of the drop. Multiple-exposure dark-field photographs were obtained, using a flash frequency equal to about twice the mean beat frequency of the spermatozoa in a particular reactivation solution. The photographs were printed at a final magnification of $\times 2000$, and wavelengths were measured along the image of the flagellum by using a piece of flexible polyethylene tubing.

With the best preparations, obtained with freshly collected spermatozoa from thealthy, fully ripe, sea urchins, the variance of beat frequency and other wave para- 
meters within a preparation was small, and selection of a representative sample fd quantitative measurements was not a problem. More commonly, the selection of spermatozoa for measurement involved value judgements about the quality of their movement. Spermatozoa swimming in straight paths, with precisely periodic beats and uniformly curved bent regions in their waveforms, tended to be favoured for inclusion in the sample measured. Consequently the sample tended to include those spermatozoa in a preparation which had the highest beat frequencies and largest bend angles and exclude many spermatozoa which had subnormal beat frequencies or bend angles. This procedure may be a valid method for obtaining parameters of 'normal' spermatozoa, but may be a source of error if the characteristics of the distribution change with important experimental variables such as ATP concentration and viscosity. This possible source of error precluded measurements at higher viscosities and ATP concentrations than were included in the results shown in this paper.

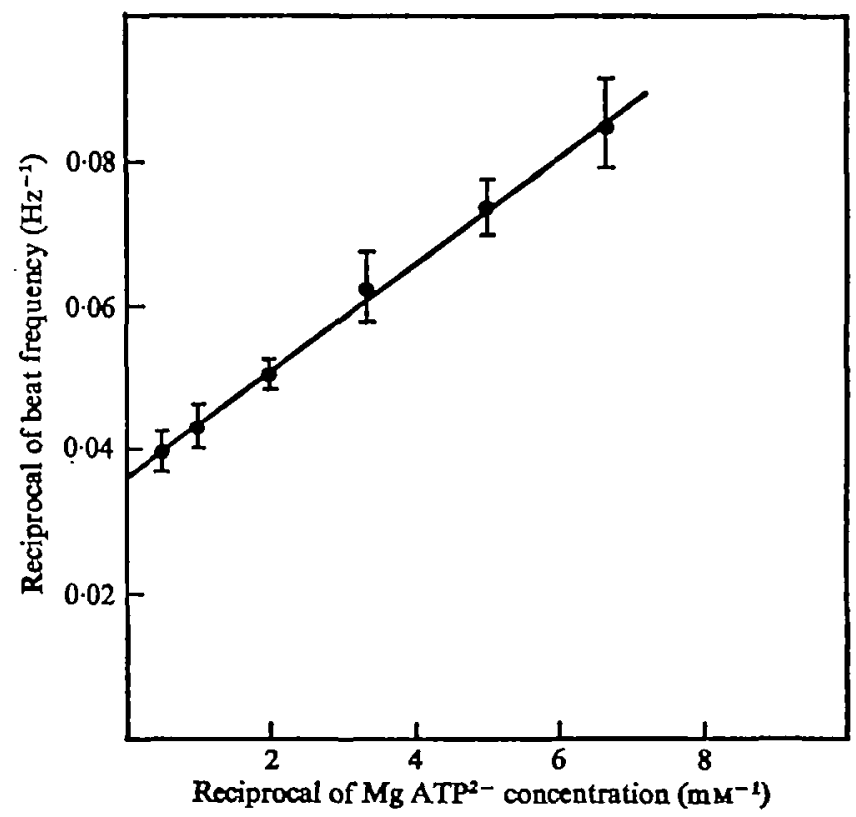

Fig. I. Double reciprocal plot of beat frequency and MgATP2- concentration from an experiment with a single sample of Lytechinus spermatozoa. Means and standard deviations are indicated for the measured beat frequencies of 27 spermatozoa at each ATP concentration.

\section{RESULTS}

\section{Effects of ATP concentration}

The beat frequency of reactivated spermatozoa increases with increasing ATP concentration in the range of $0.01-1.0 \mathrm{mM}$ (Hoffmann-Berling, 1955; Kinoshita, 1958) and levels off to a maximum frequency above I mM. This saturation effect can be described accurately by Michaelis-Menten type kinetics (Brokaw, 1967; Holwill, 1969; Gibbons \& Gibbons, 1972). Fig. I is a double reciprocal plot of beat frequency and $\mathrm{MgATP}^{2-}$ concentration over the concentration range of $0.15^{-2} \cdot 0 \mathrm{mM}$. Although there is some scatter in the measurements, the results are clearly linear, and yield 1 


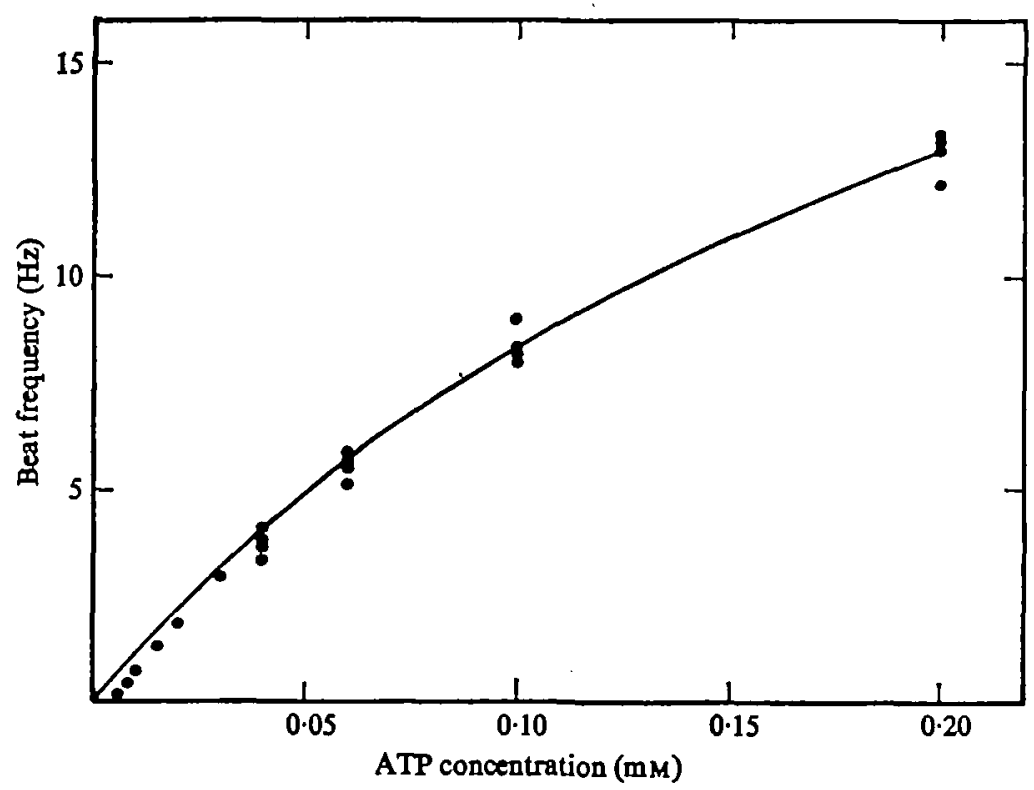

Fig. 2. Measurements of beat frequency at low ATP concentrations. The points at the four highest ATP concentrations represent data from experiments with four different sperm samples, and each point represents the mean of 20 measurements of beat frequency. The points at 0.01 and $0.02 \mathrm{mM} \mathrm{ATP}$ also represent measurements from four experiments, while the remaining points represent measurements from two experiments. Only the mean value is shown at these lower ATP concentrations, as the values from individual experiments fell too close together to be shown individually. The curve is obtained from the Michaelio-Menten equation, with $f_{\max }=27 \mathrm{~Hz}$ and $K_{m}=0.22 \mathrm{mM}$.

maximum beat frequency of $28 \mathrm{~Hz}$ and a $K_{m}$ value of $0.20 \mathrm{mM} \mathrm{MgATP}^{2-}$. This $K_{m}$ value is very close to the value obtained with Triton-extracted spermatozoa of Colobacentrotus (Gibbons \& Gibbons, 1972). Earlier experiments with glycerol-extracted Lytechinus spermatozoa (Brokaw, 1967; Brokaw \& Josslin, 1973) gave significantly higher values for $K_{m}$, about $0.5 \mathrm{mM}$ ATP in the presence of $4 \mathrm{mM}-\mathrm{MgSO}_{4}$, which corresponded to significantly lower beat frequencies at ATP concentrations of $0.2 \mathrm{~mm}$ or lower. This difference may be related to the more thorough membrane removal achieved with the Triton-extraction procedure.

Many samples of Lytechinus spermatozoa which gave normal movements at ATP concentrations of $0.2 \mathrm{mM}$ or less showed very poor motility in reactivation solutions containing I mM or more ATP. In these preparations, there were no, or very few, spermatozoa swimming stably with frequencies in the range of $25-30 \mathrm{~Hz}$. The movement was noticeably aperiodic, and many of the spermatozoa had beat frequencies in the range of $10-15 \mathrm{~Hz}$, with variable waveforms. It was difficult to find samples of Lytechinus spermatozoa which would behave consistently enough to obtain usable measurements at ATP concentrations above $0.5 \mathrm{~mm}$. The reason for this behaviour was not found. In the same reactivation solutions, Triton-extracted spermatozoa from the sea urchin, Strongylocentrotus purpuratus, routinely swam with stable frequencies of around $25 \mathrm{~Hz}$ at $1 \mathrm{mM} \mathrm{ATP}$, even after storage of the sperm sample for $24 \mathrm{~h}$.

Fig. 2 shows the results of beat frequency measurements on spermatozoa reactivated at ATP concentrations of $0.2 \mathrm{~mm}$ and below. These results are compared with a 


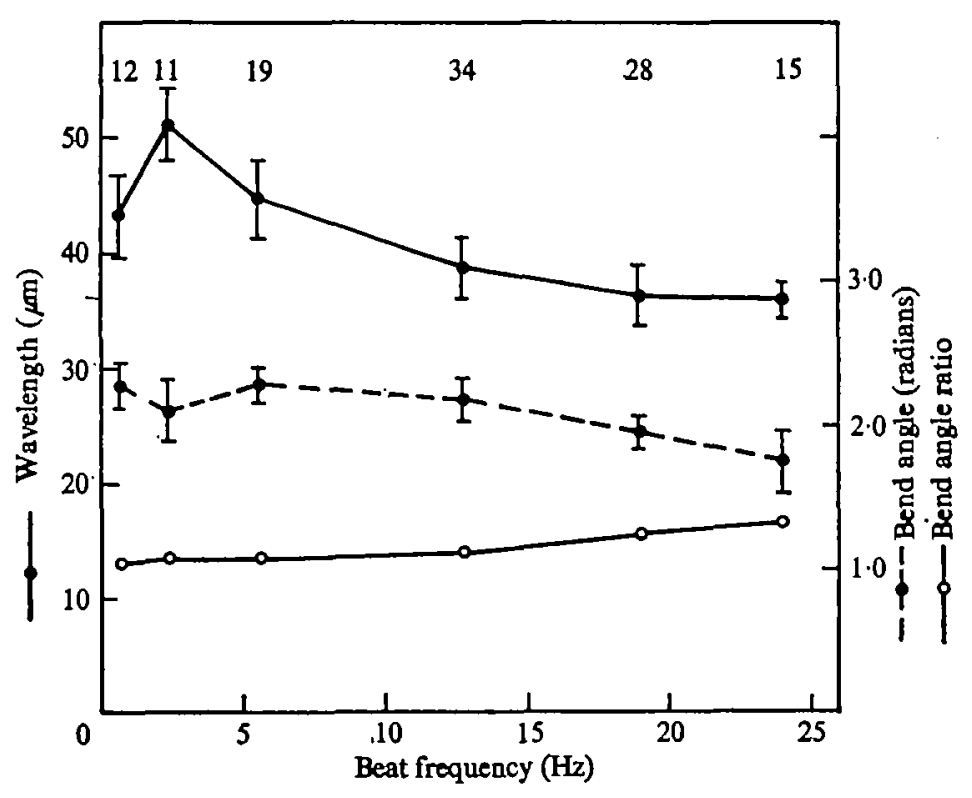

Fig. 3. Results of measurements on photographs of epermatozoa reactivated at ATP concentrations from 0.01 to $1.0 \mathrm{~mm}$. The upper two curves show measurements of wavelengths and bend angles of swimming spermatozoa. Standard deviation ranges for these measurements are shown, and the number of spermatozoa in each sample is indicated at the top of the figure. The lower curve shows the ratio of average bend angle for attached spermatozoa to average bend angle for swimming spermatozoa. Only the ratios at the three higher ATP concentrations are significantly greater than $1 \cdot 0$.

Michaelis-Menten curve which fits the data at the upper end of this ATP concentration range, and corresponds to a maximum beat frequency of $27 \mathrm{~Hz}$ and a $K_{m}$ of $0.22 \mathrm{~mm}$ ATP. As found previously with Colobocentrotus spermatozoa (Gibbons \& Gibbons, 1972), the beat frequencies of reactivated Lytechinus spermatozoa fall below the calculated Michaelis-Menten curve at low ATP concentrations (below about $0.05 \mathrm{mM})$. No movements can be observed at ATP concentrations below about $0.004-0.005 \mathrm{mM}$, and in the ATP concentration range of $0.005-0.010 \mathrm{mM}$, only a fraction of the spermatozoa in a suspension are beating (Brokaw, 196r; Gibbons \& Gibbons, 1972). In this ATP-concentration range, the bending movements do not give the impression of a regular undulatory wave, but do show bending which propagates alternately along the entire length of the flagellum. With o.0ro mM ATP, most of the spermatozoa are reactivated, and show uniform undulatory wave motion, but a few sperm samples showed no movement until the ATP concentration was raised to $0.015 \mathrm{~mm}$. Figs. IOA and B (Plate $\mathrm{r}$ ) show photographs of spermatozoa swimming in a solution containing $0.010 \mathrm{mM} \mathrm{ATP}$, where the beat frequencies were approximately $0.7 \mathrm{~Hz}$. Although some spermatozoa swim at this ATP concentration with nearly normal waveforms, as illustrated in Fig. Io B (Plate I), it is more common to find waveforms with significantly longer than normal wavelengths, as illustrated by Fig. IoA (Plate I).

Results from a series of experiments in which reactivated spermatozoa were photographed at ATP concentrations ranging from 0.012 to $1.0 \mathrm{mM}$ are shown in Fig. 3. 
These quantitative data from a relatively small number of sperm samples show at least qualitatively the behaviour which was typically observed. However, there was considerable variation between sperm samples, particularly at the ends of the ATP concentration range. The sharp decrease in wavelength between 0.025 and $0.012 \mathrm{~mm}$ ATP was commonly, but not consistently, observed - note that it does not appear in the data in Fig. 6 B. An even greater change in wavelength in this concentration range has been observed with spermatozoa from Strongylocentrotus purpuratus. In previous work with glycerinated Lytechinus spermatozoa (Brokaw \& Josslin, I973), changes in wavelength and bend angle in the concentration range of $0.11-3.0 \mathrm{mM}$ ATP (beat frequencies $4.8-24.5 \mathrm{~Hz}$ ) were observed which were similar to the changes shown in Fig. 3. However, the wavelengths generated by the symmetrically beating spermatozoa studied in the present experiments are significantly longer. Visual observations indicated that the mean bend angle continues to decrease at ATP concentrations above I mM, although a few spermatozoa can be found with normal (ca. 2 radians) bend angles even at $4 \mathrm{mM} \mathrm{MgATP}^{2-}$.

\section{Effects of increased viscosity}

Measurements of the movement parameters of reactivated spermatozoa as a function of viscosity, at various ATP concentrations, are summarized in Figs. 4-6. The data in these figures were obtained with different sperm samples at each ATP concentration, and therefore do not give as good an indication of the relationships between movement parameters and ATP concentration as the data in Figs. I-3.

As shown in Fig. 4, the effect of increased viscosity on the beat frequency of spermatozoa reactivated at high ATP concentrations is similar to its effect on live spermatozoa (Brokaw, I966). At lower ATP concentrations, the effect of viscosity diminishes, and at ATP concentrations of $0.01-0.025 \mathrm{mM}$, the frequency is almost independent of viscosity over the range of $\mathrm{x} \cdot 8-\mathrm{r} 6 \mathrm{cP}$. At higher viscosities, some decrease in beat frequency was observed at $0.0 \mathrm{rmM}$ ATP, but the number of spermatozoa swimming with stable movements was decreased, and the measured beat frequency may represent those spermatozoa which were able to generate stable movements because they had lower beat frequencies. Over the range of viscosities shown in Fig. 4, the measured frequencies fall on approximately straight lines when plotted on a double logarithmic plot, and it is convenient to use the slope of these lines as an indicator of the effect of viscosity. In Fig. 5, the data are summarized by a plot of these slopes against the beat frequency at $\mathrm{x} .8 \mathrm{cP}$, for comparison with theoretical curves which will be discussed later. The data shown in Fig. 5 for reactivated spermatozoa suggest that the slope increases towards a value near -0.3 when the beat frequency reaches a maximum value near $30 \mathrm{~Hz}$; however, the data for live spermatozoa at $2.9 .5 \mathrm{~Hz}$ have a slope of only -0.25 .

The measurements shown in Fig. 6A show that viscosity also has very little effect on the bend angle at low ATP concentrations (0.012 and $0.025 \mathrm{mM}$ ). At higher ATP concentrations, the bend angle decreases significantly at increased viscosities. There is also a large increase in the variability of the waveform, and the preparations contain spermatozoa with normal (Fig. IOD, Plate I) and much lower than normal (Fig. IoE, Plate I) bend angles. Since there was a tendency to select spermatozoa for photography with near-normal bend angles, in order to obtain accurate wavelength 


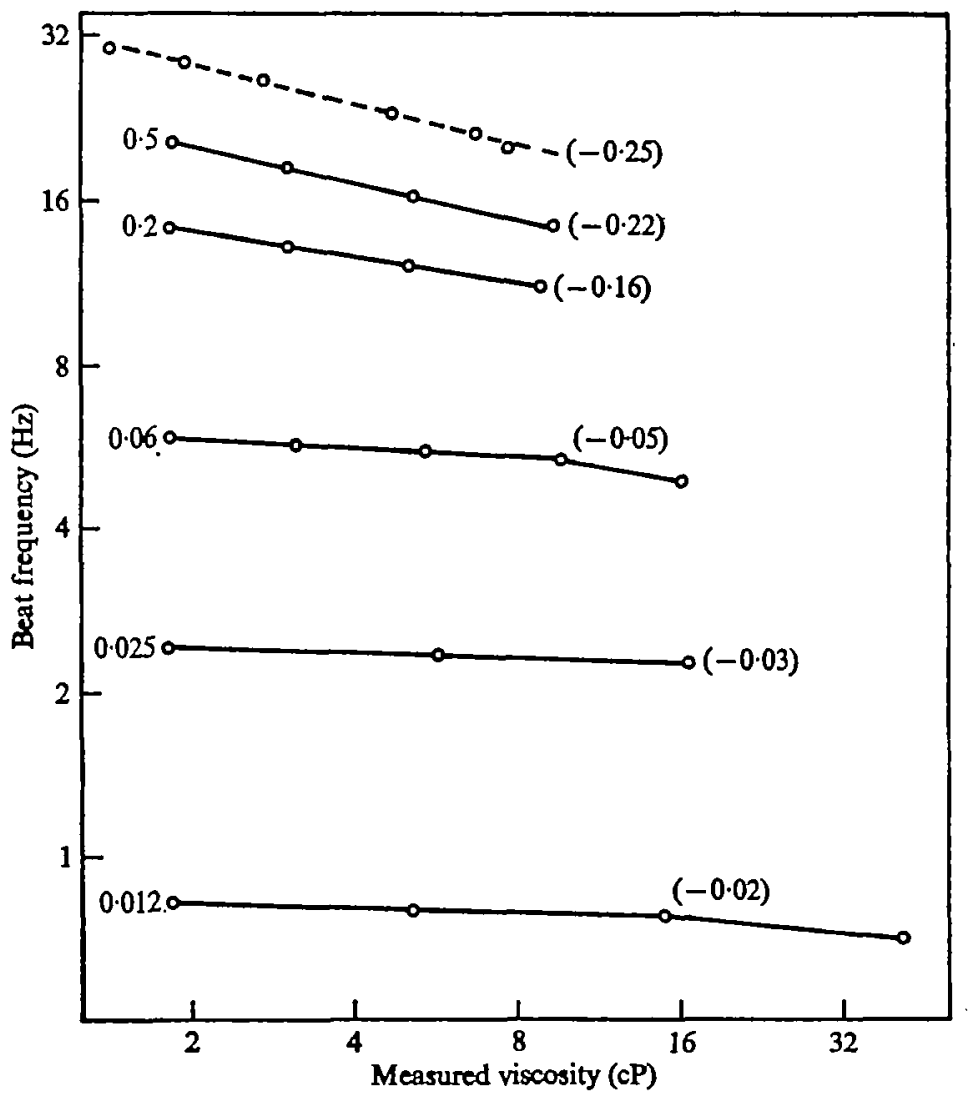

Fig. 4. Double logarithmic plot of beat frequency and viscosity for spermatozoa reactivated at various ATP concentrations. Data are also shown (dashed line) for live spermatozoa (from Brokaw, 1966). ATP concentrations in $\mathrm{mM}$ are indicated at the left end of each curve, and the slopes of the logarithm of beat frequency ve. logarithm of viscosity are indicated at the right end of each line. In each preparation examined, a mean beat frequency was determined by recording the frequency for ro-I 5 spermatozoa. The standard deviations of the frequencies recorded from one such preparation ranged up to so \% of the mean beat frequency. Each point in this figure represents the mean of 2-10 such measurements, using 2-4 different sperm samples at each ATP concentration. Standard errors for these means averaged about $2 \%$ of the mean beat frequency, which is represented by the radius of the circles used to locate the points. The largest standard error was $3 \%$ of the mean beat frequency.

measurements, the bend angles shown in Fig. 6A at high frequencies and viscosities may be too high.

In earlier work with glycerol-extracted spermatozoa from Lytechinus (Brokaw, I966), a significant increase in bend angle with increasing viscosity was observed with spermatozoa reactivated at $0.2 \mathrm{mMATP}$, but not with spermatozoa reactivated at 2.0 mM ATP. This effect was not seen in the present experiments with Tritonextracted spermatozoa, either at $0.2 \mathrm{mM} \mathrm{ATP}$ or at $0.06 \mathrm{mM} \mathrm{ATP}$, where the beat frequencies are more comparable to those obtained with glycerol-extracted spermatozoa at $0.2 \mathrm{mM}$ ATP.

The measurements shown in Fig. 6B, and the photographs of typical waveforms shown in Fig. 10 C-I (Plate I), show that, in contrast to the effects on bend angle and beat frequency, the effect of viscosity on wavelength remains nearly constant over the 


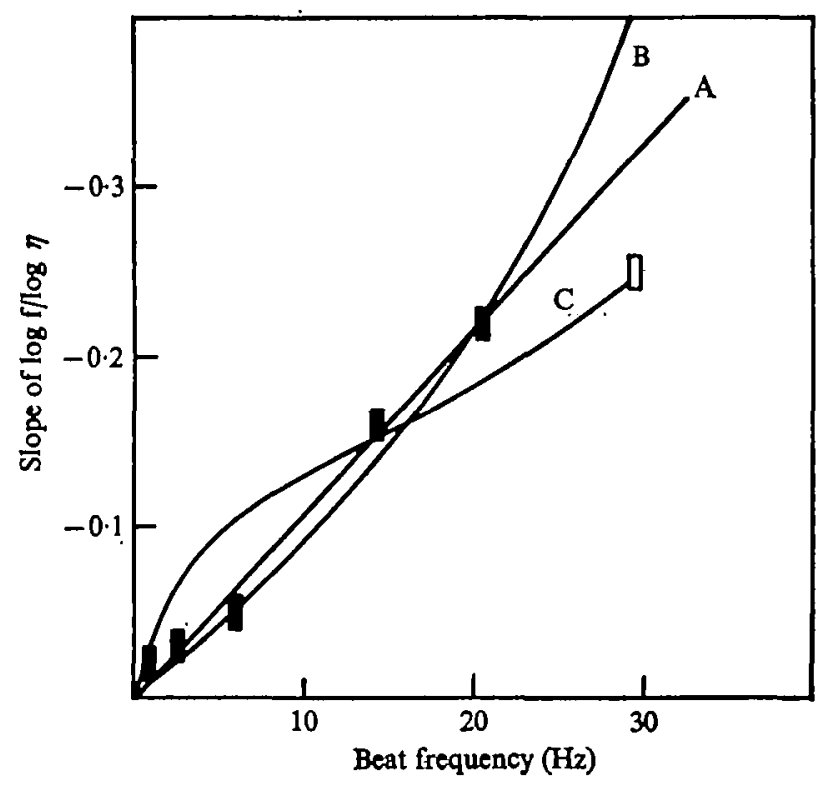

Fig. 5. Slopes of $\log$ frequency ve. $\log$ viscosity for the data in Fig. 4, plotted against the value of beat frequency at a viscosity of $1.8 \mathrm{cP}$. Solid bars represent reactivated spermatozoa; the open bar represents live opermatozoa. Error bars of $+/-0.01$ slope units and $+1-0.25$ beat frequency units $\left(\mathrm{Hz}_{2}\right)$ have been arbitrarily selected as a reminder that there is some uncertainty in the values derived from Fig. 4. The theoretical curves are identified in the Discussion.

ATP concentration range from 0.5 to $0.025 \mathrm{~mm}$ and is similar to the effect with live spermatozoa. Only at $0.012 \mathrm{~mm}$ ATP is there a clear decrease in the sensitivity of wavelength to increased viscosity. At this ATP concentration there is still a significant effect of viscosity on wavelength, even though the effects of viscosity on bend angle and beat frequency at this ATP concentration are minimal.

\section{Relationship between bend angle and beat frequency}

Live spermatozoa which become attached by their heads to a microscope slide or coverglass regularly show an increase in bend angle, which may be accompanied by small decreases in beat frequency and wavelength (Brokaw, 1965; Gibbons, 1974). With reactivated spermatozoa, this effect is seen at high ATP concentrations, but disappears at low ATP concentrations (Fig. 3, bottom curve). The effect of head attachment can be increased by increasing the viscosity of the reactivation solution, and at higher ATP concentrations and viscosities, large angle bending patterns (Fig. IOF, Plate I) are regularly obtained with attached spermatozoa. These effects offer an opportunity for examination of the correlation between bend angle and beat frequency in an attempt to determine whether interfilament shear rate is the ratelimited variable which determines flagellar beat frequency at low ATP concentrations. If this is the case, the product of beat frequency and bend angle, which is proportional to shear rate, might be expected to show less variation than is shown by the bend angle, and this effect ought to be more pronounced at low ATP concentrations than at higher ATP concentrations where viscosity becomes a rate-limiting factor.

Visual observations of beat frequency and bend angle were made on individual 


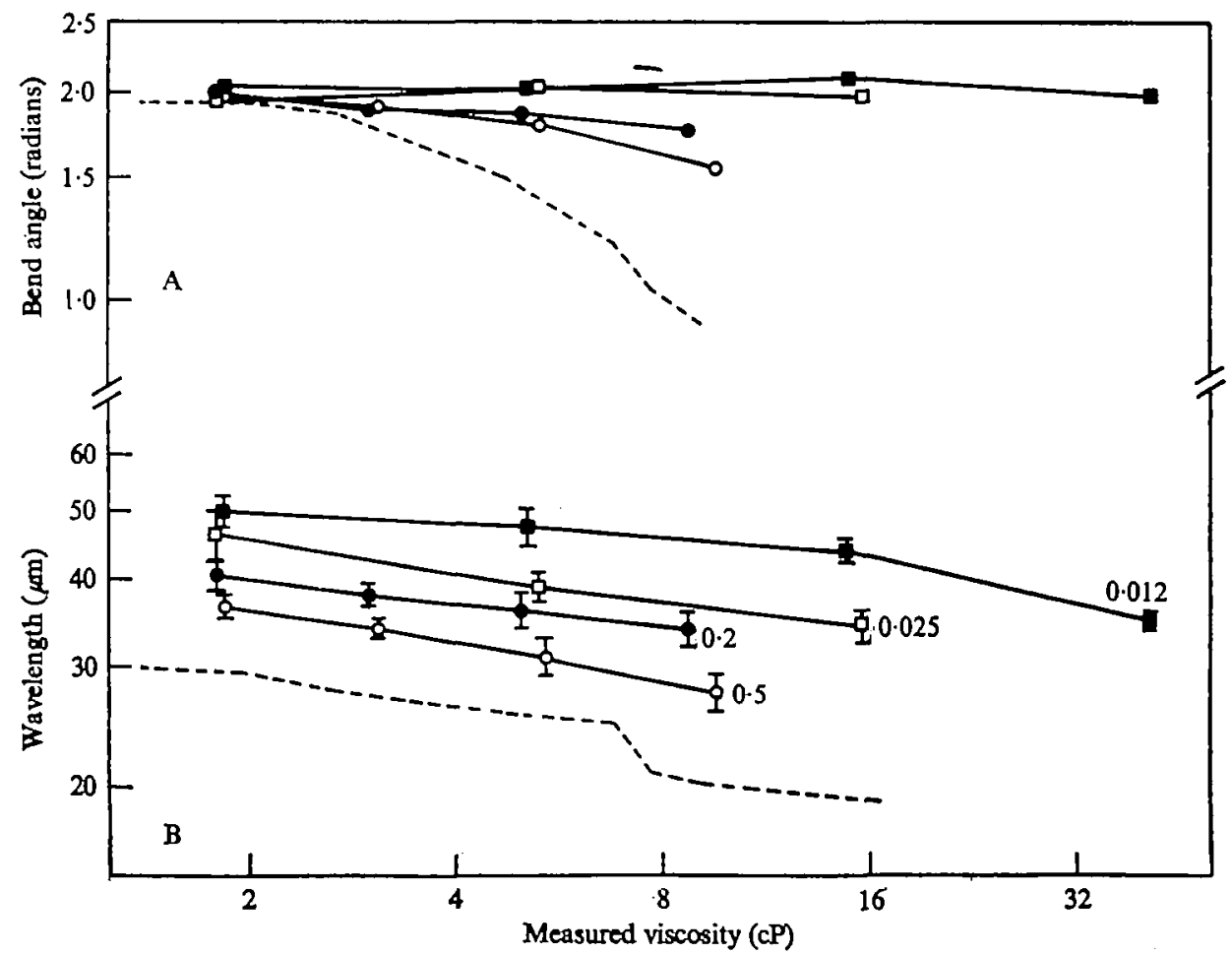

Fig.6. Double logarithmic plots ahowing the effects of viscosity on bend angles and wavelengths of apermatozoa reactivated at various ATP concentrations. Data are also shown (dashed lines) for live spermatozoa (from Brokaw ( 1966 ) and new measurements of bend angles on the same photographs). ATP concentrations are identified by the symbols and the numbers at the right end of the wavelength curves. The points at $0.012 \mathrm{mM}$ ATP were obtained with a single sperm sample, and each point represents measurements of approximately six spermatozoa. The points at the higher ATP concentrations represent measurements of approximately 20 spermatozoa from 2-4 different sperm samples Standard deviations for the bend angle measurements ranged from 0.07 to 0.24 radians, except for the highest viscosity point at $0.5 \mathrm{mM} \mathrm{ATP}$, where the sample exhibited a wide range of variation of bend angle and the standard deviation for the measured bend angles was 0.47 radians.

spermatozoa, and an effort was made to select spermatozoa at the extremes of the range of bend angles exhibited by the sperm sample. Observations were made at two ATP concentrations: in reactivation solutions containing $0.24 \mathrm{mM}$ ATP, $0.70 \mathrm{~mm}$ $\mathrm{MgSO}_{4}$ and $0.3 \%$ methyl cellulose, with viscosities of about $\mathrm{ro} \mathrm{cP}$, and in reactivation solutions containing $0.024 \mathrm{~mm}$ ATP, $0.52 \mathrm{~mm}-\mathrm{MgSO}_{4}$ and $0.5 \%$ methyl cellulose, with viscosities of about $30 \mathrm{cP}$. Observations on attached spermatozoa were restricted to those which had the sperm head rigidly attached to the microscope slide, so that no rotation or oscillation of the sperm head was seen. The results are summarized in Fig. 7 .

There is no significant difference between the results obtained at the two ATP concentrations. At both ATP concentrations the samples of attached spermatozoa had mean bend angles of 2.8 radians and the samples of swimming spermatozoa had mean bend angles of $I \cdot 9$ radians. The samples of attached spermatozoa had mean beat frequencies which were lower than the mean beat frequencies of the swimming sperm samples, by $17 \%$ at $0.24 \mathrm{~mm} \mathrm{ATP}$ and $16 \%$ at $0.024 \mathrm{mM} \mathrm{ATP}$. This decrease if 


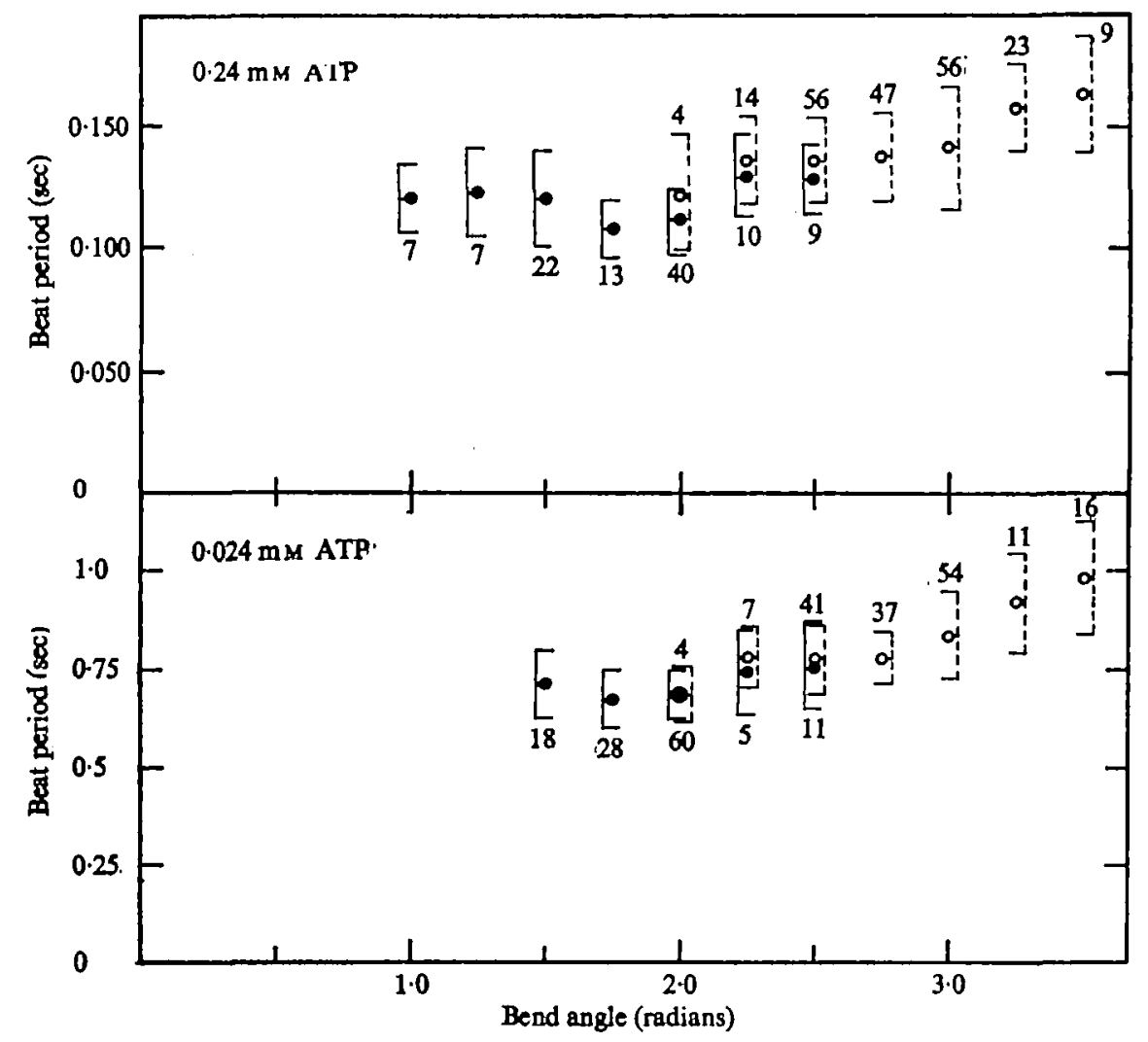

Fig. 7. Measured values of beat period and bend angle for swimming (solid circles) and attached (open circles) spermatozoa. The number of spermatozoa measured, and the standard deviation of the measured values of beat period, are shown for each bend angle group.

beat frequency is probably a secondary consequence of the increase in bend angle in the attached sperm samples. At a particular bend angle, the differences in mean beat frequency between swimming and attached sperm samples were small and probably not significant.

If shear rate is a constant parameter characteristic of the spermatozoa in a sample at a particular ATP concentration, the results should lie on a straight line through the origin when beat period is plotted against bend angle, as in Fig. 7. The swimming sperm samples show a weak beat frequency maximum near the mean bend angle of I.9 radians, but otherwise show no significant correlation between beat period and bend angle. The expected correlation is seen at bend angles of 3 radians or more, where the beat frequencies of attached spermatozoa decrease sufficiently at high values of the bend angle to maintain constant shear rate. For bend angles in the range of 2-3 radians, the correlation is weaker, although it is sufficient to account for the difference between the mean beat frequencies of swimming and attached spermatozoa.

Although these observations fail to provide strong evidence for the possibility that shear rate is the important rate-limited variable at low ATP concentrations, they are not definitive, as the expected correlation could easily be obscured by variations in hear rate within the population examined. 


\section{DISCUSSION}

The main features of the symmetrical flagellar bending waves observed in these experiments can be described by three parameters - wavelength (measured along the flagellum), bend angle, and beat frequency. As noted previously by Gibbons (1974), flagellar wave parameters appear to be regulated independently, with only secondorder interactions observed between them.

ATP concentration is a major factor determining the beat frequency of reactivated flagella; changes in beat frequency of two orders of magnitude can be observed over the ATP concentration range of 0.005 to $4 \mathrm{~mm}$. On the other hand, ATP concentration changes, particularly over the range of $0 \cdot 05-1 \cdot 0 \mathrm{mM}$, have rather small effects on wavelength and bend angle, which may be a secondary result of changes in viscous forces resulting from changes in beat frequency, rather than a direct effect of ATP on these wave parameters.

The effects of changes in viscosity on flagellar wave parameters are small compared to the effect of ATP concentration on beat frequency, and over most of the range of ATP concentrations viscosity changes cause changes of similar magnitude in all three wave parameters. One theoretical model for the generation of flagellar bending waves which has been studied in detail incorporates an internal viscous bending resistance to stabilize the wavelength of its motion (Brokaw, $1972 a$ ). This model then predicts, without specification of any additional parameters, that frequency and wavelength should each be inversely proportional to the $4^{\text {th }}$ root of the external fluid viscosity, giving a slope of -0.25 on a double logarithmic plot against viscosity (Brokaw, $1972 b, c)$. This slope is approached by the data on beat frequency of reactivated spermatozoa at high ATP concentrations (Fig. 4), but the wavelength data (Fig. 6B) show consistently lower slopes.

The inclusion of an internal viscosity in this model to regulate the wavelength provides an example of a wavelength-regulating mechanism which might be expected to be independent of the effects of ATP concentration. However, the internal viscosity hypothesis is objectionable because it increases, to a possibly unrealistic degree, the energy requirements for the active processes which generate flagellar movements (cf. Brokaw \& Gibbons, 1975). A more satisfactory explanation for wavelength regulation in flagella has not yet been developed.

A direct determination of beat frequency by intrinsic kinetic properties of the mechanochemical system within flagella has been assumed in previous studies of the effect of ATP concentration on flagellar beat frequency (Holwill, 1969) and is consistent with indications of a close coupling between beat frequency and ATP dephosphorylation (Brokaw \& Benedict, I968 $a, b$; Gibbons \& Gibbons, 1972). On the other hand, to explain the influence of viscosity on beat frequency, most theoretical models for flagellar wave propagation have emphasized moment balance between viscous, elastic, and active bending moments (Machin, 1958; Rikmenspoel, I97I; Brokaw, 1971, 1972a).

The effects of ATP concentration on the relationship between beat frequency and viscosity can be summarized by saying that at low ATP concentrations the beat frequency is determined by some intrinsic property of the active sliding process which is sensitive to ATP concentration, and viscous resistances are insignificant. At higher 
RTP concentrations the ATP-concentration-dependent step in the beat cycle is no longer rate limiting, and viscous forces become predominant in determining the beat frequency. This behaviour can be modelled in a purely formal way by assuming that beat frequency is determined by the overall rate of a two-step reaction sequence or reaction cycle, in which one step is sensitive to viscosity and the other step is sensitive to ATP concentration. The beat frequency, $f$, will then be governed by the following relationship:

$$
\mathrm{I} / f=\mathrm{I} / r_{1}+\mathrm{I} / r_{2},
$$

where $r_{1}$ is a function of viscosity and $r_{2}$ is a function of ATP concentration. If $r_{1}$ is only a function of viscosity, and if $r_{2}$ is directly proportional to ATP concentration, then at any constant viscosity, equation (I) will give a relationship between beat frequency and ATP concentration equivalent to Michaelis-Menten kinetics, in agreement with experimental results with flagella. In the simplest case, $r_{1}$ might be inversely proportional to viscosity. However, if other parameters of flagellar movement change with viscosity, the 'effective viscosity' experienced by active mechanochemical elements in the flagellum will change. In the model for small amplitude wave movements along an infinite filament considered by Brokaw (1971, 1972 $b$ ), the 'effective viscosity' experienced by a generator of active shear moment will be proportional to $\eta \lambda^{3}$, where $\eta$ is the viscosity and $\lambda$ is the wavelength (cf. equation 6 of Brokaw, 1972b). Since this is a small amplitude model, it includes the assumption that both active shear moment and viscous bending moment are linear functions of the curvature of the flagellum, so that variations in bend angle do not influence the 'effective viscosity'. If the wavelength is regulated by an internal viscous bending resistance, it will be proportional to $\eta^{-0.25}$ (Brokaw, $1972 b$ ), and the 'effective viscosity' will therefore be proportional to $\eta^{\gamma}$, where $\gamma=\mathrm{I}-3(0.25)=0.25$. Equation (I) can then be rewritten to explicitly include the effects of viscosity and ATP concentration:

$$
\mathbf{I} / f=c_{1} \eta^{\gamma}+c_{2} / C,
$$

where $C$ represents the concentration of ATP (or $\mathrm{MgATP}^{2-}$ ) and $c_{1}$ and $c_{2}$ are constants. Examination of the relationship between beat frequency and viscosity at various ATP concentrations predicted by equation (2) by construction of a double logarithmic plot similar to Fig. 4 indicates that equation (2) provides a reasonably accurate description of the experimental results shown in Fig. 4, as well as the relationship between frequency and ATP concentration at constant viscosity. By differentiation of equation (2),

$$
\frac{d \ln f}{d \ln \eta}=-c_{1} \gamma f \eta^{\gamma},
$$

indicating that a plot of the $\log f$ vs. $\log \eta$ slope against beat frequency, as in Fig. 5 should give a straight line (curve $A$ in Fig. 5) and that $-\gamma$ will be equal to twice the slope obtained at an ATP concentration equal to $K_{m}$. The experimental data therefore suggest that $\gamma$ should have a value of approximately $0.3 \mathrm{I}$, rather than 0.25 . The data in Fig. 6B suggest that the change in wavelength with viscosity is less than predicted by the internal viscosity hypothesis. If the slope of $\log \lambda$ vs. $\log \eta$ is about -0.15 , which may be more consistent with the data in Fig. 6B, the value of $\gamma$ would be expected to be about 0.55 . 
Although this model is in good agreement with the experimental data on effects 0 ATP concentration and viscosity on flagellar beat frequencies, it does not provide much insight into the active mechanisms in flagella. Some more specific models which may be more informative are considered in the following two sections.

\section{Effects of flagellar elasticity on the regulation of beat frequency}

In the flagellar model developed to incorporate a feedback control of active shear moment by curvature, beat frequency is determined by the balance between active and viscous bending moments (Brokaw, 1971, r972a,b,c). Elastic resistances were included in this model as a possible means for regulation of bend angle, but were kept small enough to make a negligible contribution to the determination of beat frequency by active and viscous bending moments.

Estimates of the elastic properties of flagella suggest that elastic resistance in flagella may be a significant component of the moment balance equation at normal beat frequencies of about $30 \mathrm{~Hz}$ (Lindemann, Rudd \& Rikmenspoel, r973; Brokaw, 1975a). Since viscous bending moments, but not elastic bending moments, are proportional to velocities of movement, the elastic resistances will become very much larger than the viscous resistances when beat frequencies are in the neighbourhood of I $\mathrm{Hz}$. In terms of a model which balances active, viscous and elastic bending moments, the active shear moment generated at low beat frequencies must follow the curvature with a phase lag of nearly beat cycle, in order for most of the active bending moment to occur in phase with the bending moment resulting from elastic resistances. Only a small fraction of the active bending moment will then occur in phase with the bending moment resulting from viscous resistances. In the models developed previously (Brokaw, 1971, 1972 $a$ ) a small time delay between curvature and active shear moment was introduced to provide a component of active moment in phase with elastic moments. If this model is extended to operation at low beat frequencies where the movement is dominated by elastic resistances, the time delay becomes the principal determinant of beat frequency. If the time delay causes a phase lag of nearly beat cycle, a small change in beat frequency will be able to cause a large change in the magnitude of the active moment component in phase with viscous moments, so that this flagellar model could accommodate to changes in external viscosity with little change in beat frequency.

A quantitative development of these ideas can be carried out for small amplitude wave propagation along an infinite filament by an extension of the earlier analysis (Brokaw, $1972 b$ ). The 'effective viscosity' experienced by a generator of active shear moment then becomes $\eta^{\gamma} / \cos 2 \pi f \tau$, where $2 \pi f \tau$ is the phase angle between curvature and active shear moment, generated by a time delay, $\tau$. The time delay must vary considerably with ATP concentration, in order to keep $2 \pi f \tau$ within the permissible range of $0-1 \pi$. If the effect of ATP concentration is expressed solely by its effect on a rate constant which determines $\tau$, and $\mathrm{r} / \tau$ is assumed to be proportional to ATP concentration, the frequency will be determined by

$$
\mathrm{r} / f=c_{1} \eta^{\gamma} / \cos 2 \pi f \tau+c_{2} \text {. }
$$

Equation (4) also gives a system where beat frequency, and the slope of $\log f$ vs. $\log \eta$, increase towards maximum values at high ATP concentrations. However, the shapeg 


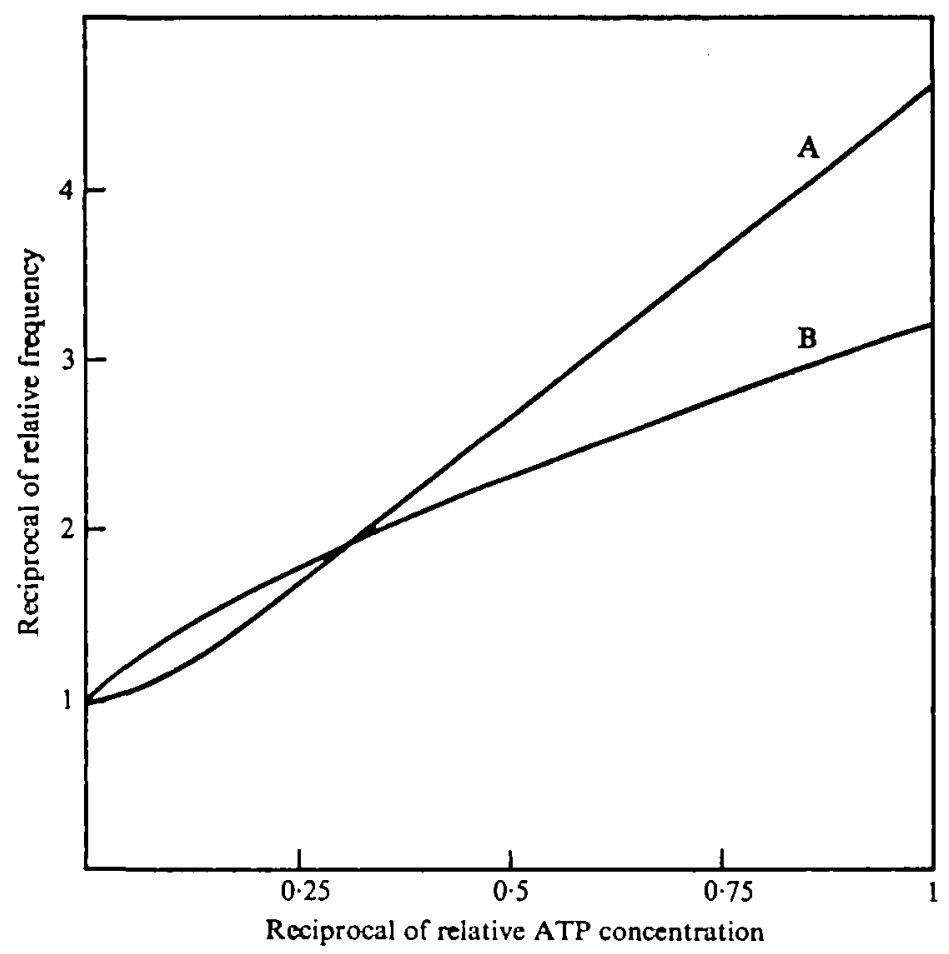

Fig. 8. Double reciprocal plots of beat frequency and ATP concentration for a model in which elastic resistances become dominant at low ATP concentrations (curve A) and for a model in which shear rate is related to shear moment by an equation similar to Hill's equation for muscle (curve B).

of the relationships are different, as illustrated by curve B in Fig. 5 and by curve A in Fig. 8. Curve B in Fig. 5 is obtained by differentiation of equation (4) to give

$$
\frac{d \ln f}{d \ln \eta}=\frac{-\gamma}{\mathrm{I}+2 \pi f \tau \tan 2 \pi f \tau} \text {. }
$$

A reasonably close fit to the experimental points in Fig. 5 can be obtained with $f_{\max }=30$ and $\gamma=0.42$, and this higher value of $\gamma$ might be more consistent with the wavelength vs. viscosity data in Fig. $6 \mathrm{~B}$. However, the relationship between beat frequency and ATP concentration shown by curve A in Fig. 8 does not agree well with the experimental observations shown in Fig. I.

With this model, a maximum beat frequency is approached as the viscous component of active shear moment approaches the total active shear moment when the phase lag between curvature and active shear moment becomes small at high values of $\mathrm{r} / \boldsymbol{\tau}$. At the same time, the active moment component in phase with elastic resistances will become small. This effect might provide a basis for explaining the decrease in bend angle which occurs at high ATP concentrations, if bend angle is determined by a balance between active and elastic bending moments, as in the models examined by Brokaw (1972a,c). 


\section{Effects of shear rate on the active shear moment}

Flagellar bending is now believed to be generated by an active sliding process occurring between the peripheral microtubules of the axoneme and resulting from an ATP-driven mechanochemical cycle involving attachment and detachment of the dynein arms. Ideas about the functioning of the dynein arms have developed from ideas about the functioning of the HMM-cross-bridges which are believed to cause active sliding between actin and myosin filaments in striated muscle. The models formulated to describe the functioning of these cross-bridges are usually such that the force generated by a cross-bridge decreases when sliding occurs, and are therefore consistent with the observed force-velocity behaviour of muscle (Hill's equation). Similar behaviour might be expected for cross-bridges in flagella, although experimental measurements have not yet given strong support for the existence of shear moment-shear rate behaviour in flagella analogous to the force-velocity behaviour of muscle (Brokaw, $1975^{b}$ ).

A quantitative evaluation of a model incorporating a Hill's equation relationship between shear moment and shear rate can be carried out with the same assumptions made in the previous section, with beat frequency determined by an 'effective viscosity':

$$
\mathrm{x} / f=c_{1} \eta^{\gamma}\left(\mathrm{I}+f \theta / a V_{0}\right) /\left(\mathrm{I}-f \theta / V_{0}\right) .
$$

In equation (6), $\theta$ represents a measure of bend angle, so that $f \theta$ is proportional to shear rate. Since the experimentally observed variations in bend angle over the range of interest are small, $\theta$ will be assumed to be constant in further analysis of equation (6). $V_{0}$ represents the maximum shear rate of the sliding filament system. In muscle, $V_{0}$, but not maximum isometric tension, is strongly dependent on ATP concentration (Takahashi et al. 1965; Godt, 1974), so the situation where $V_{0}$ is directly proportional to ATP concentration will be examined. The constant $a$, which comes from Hill's equation, will be given a value of 0.25 , based on values measured in frog skeletal muscle (cf. Hill, 197 ). The predictions of this model are shown by curve $\mathrm{C}$ of Fig. 5, with $\gamma=0.25$ and $f_{\max }=30$, and by curve $\mathrm{B}$ of Fig. 8. This model also predicts an increase in both beat frequency and slope towards a maximum value at high ATP concentrations, as a result of the linear effect of ATP concentration on maximum shear rate, $V_{0}$. However, it is quantitatively less satisfactory than either of the previous models.

The idea that beat frequency is determined indirectly as a result of an effect of ATP concentration on maximum shear rate is attractive, because shear rate would be expected to be a fundamental variable of an active sliding system involving crossbridge behaviour similar to that suggested for muscle. However, if this is the case, some indications of an inverse relationship between beat frequency and bend angle might be expected to be observed, especially at low ATP concentrations where shear rate, rather than a balance of moments, might be the major determinant of beat frequency. The experiments summarized by Fig. 7 were carried out in an effort to find such a relationship, but they failed to provide significant support for the idea that shear rate is a more fundamental variable than beat frequency. 


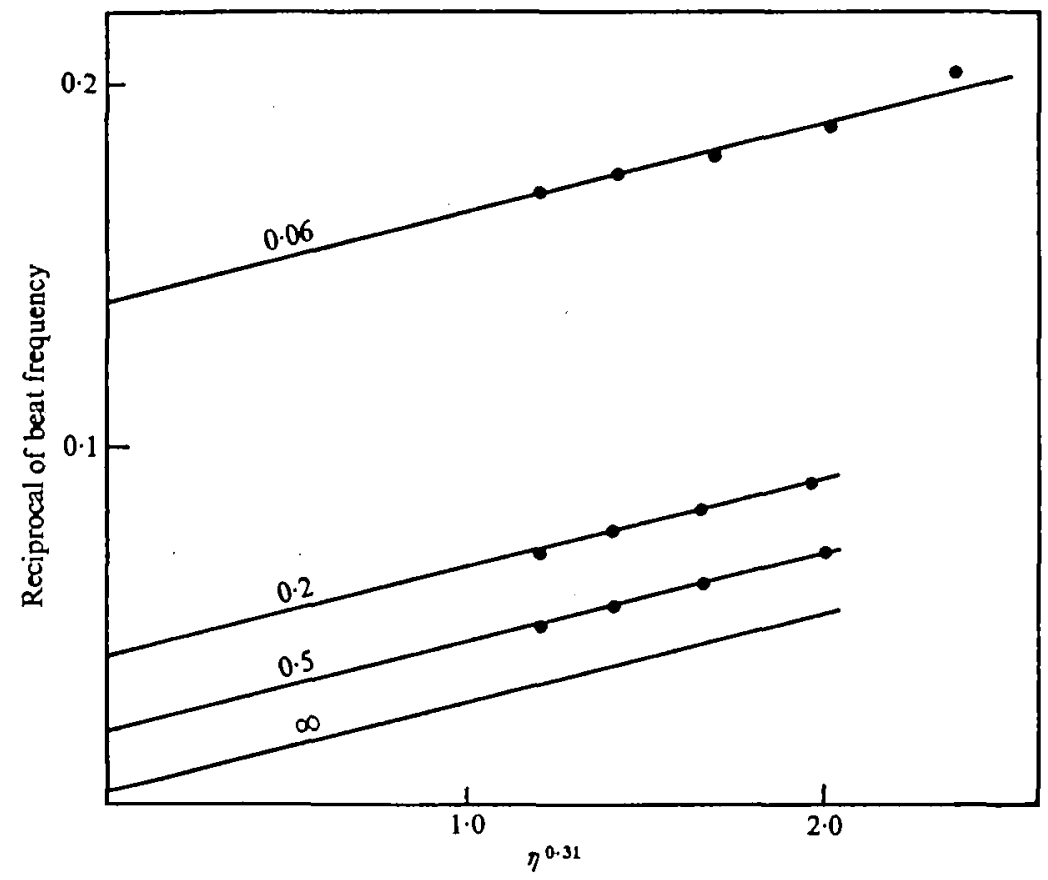

Fig. 9. Replotting of the experimental data in Fig. 4 as $1 / f$ v8. $\eta^{\gamma}$, with $\gamma=0.3 \mathrm{I}$, for comparison with the linear relationship predicted by equation (2).

\section{Evaluation}

Both of the more specific models discussed in the two preceding sections are based on factors which could reasonably be expected to influence the active mechanochemical process in flagella. Although both models offer explanations for the increase in beat frequency and the slope of $\log f$ vs. $\log \eta$ towards maximum values at high ATP concentrations, neither appears to give a satisfactory quantitative agreement with the experimental results. In particular, the close agreement of the beat frequency vs. ATP concentration data, such as that shown in Fig. I, with a simple MichaelisMenten type of saturation process suggests that beat frequency is determined by ATP concentration in a more direct manner than suggested by either of these models. The formal model represented by equation (2) therefore gives better agreement with the experimental results. Further examination of this model may be worthwhile.

Equation (2) does not define a linear relationship between $\log f$ and $\log \eta$, but over a short range of viscosities the relationship is sufficiently close to linear to fit the results shown in Fig. 4. A better comparison between these results and equation (2) is shown in Fig. 9, where $\mathrm{x} / \mathrm{f}$ is plotted against $\eta^{\gamma}$, using a value of $0.3 \mathrm{I}$ for $\gamma$. In agreement with equation (2), the results lie on a family of parallel lines. A family of parallel lines is also obtained with $\gamma=0.25$, but with $\gamma=0.5$ the points fall on clearly non-linear curves. The lowermost line in Fig. 9 is drawn with, the same slope through a point corresponding to a maximum frequency of $30 \mathrm{~Hz}$ at $\mathrm{I} \cdot 8 \mathrm{cP}$. When extrapolated to $O$ viscosity, this line intercepts the $\mathrm{I} / f$ ordinate very close to the origin. A $\circ$ intercept would mean that there are no additional steps in the frequencydetermining reaction cycle, other than the two identified in equation (2). The 
experimental results therefore suggest that in flagella beating in the normal range of viscosities and ATP concentrations, any other steps in the reaction cycle are very fast compared to the two steps identified in equation (2).

Since the first term in equation (2) is sensitive to the wavelength, changes in wavelength with ATP concentration might cause deviations from behaviour of MichaelisMenten type at constant viscosity. However, as the results in Fig. 3 indicate, changes in wavelength with ATP concentration are small at high ATP concentrations. At lower ATP concentrations where changes in wavelength are more significant, the first term in equation (2) is small relative to the ATP concentration-dependent term.

It is probably not worth while to attempt any further comparison between the experimental results and small-amplitude models for the control of flagellar wave parameters. Analysis of the large amplitude behaviour of a model satisfying equation (2) will be required for full interpretation of these experimental results. This analysis falls beyond the scope of the present paper.

This work was supported in part by a grant from the United States Public Health Service (GM I87 I I). I thank Tom Simonick for diligent assistance with the experiments, and Dr Ian Gibbons for insightful comments and for communication of results prior to publication.

\section{REFERENCES}

Broksw, C. J. (I96I). Movement and nucleoside polyphosphatase activity of isolated flagella from Polytoma uvella. Expl Cell Res. 22, $151-62$.

Brokaw, C. J. (1963). Movement of the flagella of Polytoma woella. Y. exp. Biol. 40, 149-56.

Brokaw, C. J. (1965). Non-sinusoidal bending waves of sperm flagella. J. exp. Biol. 43, 155-69.

Brokaw, C. J. (1 966). Effects of increased viscosity on the movements of some invertebrate spermatozoa. F. exp. Biol. 45, 113-39.

BrokAw, C. J. (1967). Adenosine triphosphate usage by flagella. Science, N.Y. 156, 76-8.

Brokaw, C. J. (1970). Bending moments in free-swimming flagella. J. exp. Biol. 53, 445-64.

Brokaw, C. J. (197I). Bend propagation by a sliding filament model of flagella. Y. exp. Biol. 55, 289-304.

BrokAw, C. J. (I $972 a$ ). Computer simulation of flagellar movement. I. Demonstration of stable bend propagation and bend initiation by the sliding filament model. Biophys. Y. 12, 564-86.

Brokaw, C. J. ( $1972 b$ ). Viscous resistances in flagella: Analysis of small amplitude motion. 7. Mechanochem. Cell Mot. r, I5I-5.

BrokAw, C. J. (1972c). Computer simulation of flagellar movement. II. Influence of external viscosity on movement of the siding filament model. J. Mechanochem. Cell Mot. I, 203-I I.

Brokaw, C. J. (1974). Movement of reactivated flagella at low ATP concentration. J. Cell Biol. 63, 38A.

BrokAw, C. J. (1975a). Spermatozoan motility: A biophysical survey. In The Biology of the Male Gamete (ed. J. G. Duckett and P. A. Racey). Biol. Y. Linnean Sac. 6 (supplement no. I) (in the Press).

Brokaw, C. J. (1975 b). Cross-bridge behavior in a sliding filament model for flagella. In Molecules and Cell Movement (ed. S. Inoue and R. Stevens), Raven Press. (In the Press.)

BROKAw, C. J. \& BRNEDICT, B. (1968a). Mechanochemical coupling in flagella. I. Movement-dependent dephosphorylation of ATP by glycerinated apermatozoa. Archs Biachem. Biophys. 125, 770-8.

BrokAw, C. J. \& BENEDICT, B. (I968b). Mechanochemical coupling in flagella. II. Effects of viscosity and thiourea on metabolism and motility of Ciona spermatozoa. F. gen. Physiol. 52, 283-99.

Broksw, C. J. \& GrbBons, I. R. (1975). Mechanisms of movement in flagella and cilia. Symposium on Sroimming and Flying in Nature (ed. T. Wu, C. Brennan and C. Brokaw.) Plenum Press (in the Press).

BrokAw, C. J. \& JossLIN, R. (1973). Maintenance of constant wave parameters by sperm flagella at reduced beat frequencies. J. exp. Biol. 59, 617-28.

Broksw, C. J., Josstin, R. \& Bobrow, L. (1974). Calcium ion regulation of flagellar beat symmetry in reactivated sea urchin spermatozoa. Biochem. biophys. Res. Commun. 58, 795-800.

Gibeons, B. H. \& Gibsons, I. R. (1972). Flagellar movement and adenosine triphosphatase activity in sea urchin sperm extracted with Triton X-100. 9. Cell Biol. 54, 75-97.

GIBBons, I. R. (1974). Mechanisms of flagellar motility. In The Functional Anatomy of the Spermatosoon (ed. B. A. Afzelius), pp. 127-40. Orford: Pergamon Press.

GodT, R. E. (1974). Calcium-activated tension of akinned muscle fibers of the frog. Dependence on magnesium adenosine triphosphate concentration. J. gen. Physiol. 63, 722-39. 

Fournal of Experimental Biology, Vol. 62, No. 3

Fig. 10, Plate I
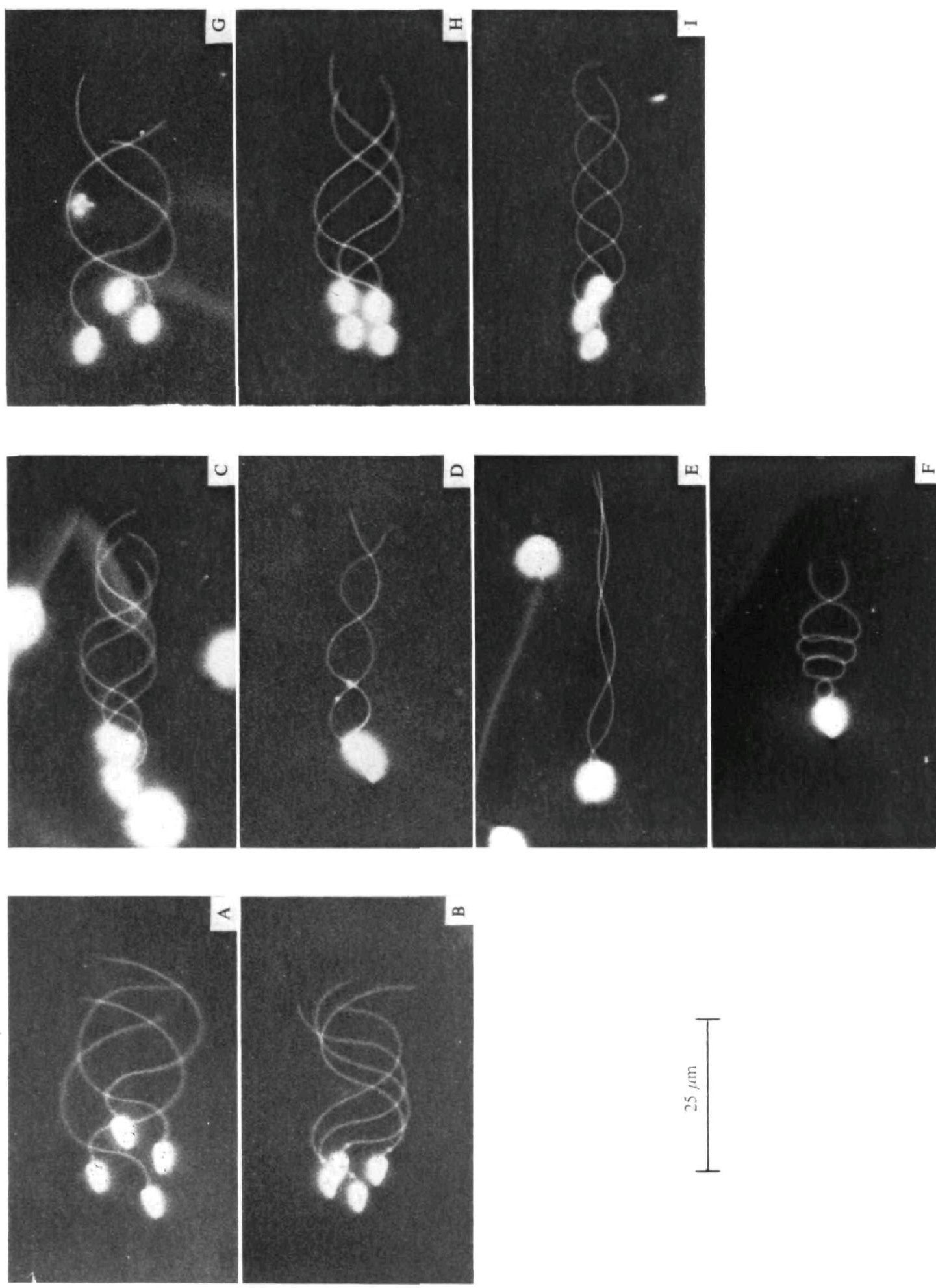

C. J. BROKAW

(Facing p. 719.) 
Ilu, A. V. (1970). First and Last Experiments in Muscle Mechanics. Cambridge University Press.

Hofmanan-Berline, H. (1955). Geisselmodelle und Adenosintriphosphate (ATP). Biochim. Biophys. Acta 16, 146-54.

HolwILl, M. E. J. (1969). Kinetic studies of the flagellar movement of sea urchin spermatozoa. $\mathcal{F}$. exp. Biol. 50, 203-22.

Kinoshita, S. (1958). The mode of action of metal-chelating substances on sperm motility in some marine forms as shown by glycerol-extracted sperm models. Y. Fac. Sci. Tokyo Univ. (Sect. 4), 8, $219-28$.

Lindemann, C. B., Rudd, W. G. \& Rikmmnspozi, R. (I973). The stiffness of the flagella of impaled bull sperm. Biophys. F. 13, 437-48.

Machin, K. E. (1958). Wave propagation along flagella. F. exp. Biol. 35, 796-806.

Rikmenspoes, R. (1971). Contractile mechanisms in flagella. Biophys. $\mathcal{Y}$. Ir, 446-63.

SAR, J.-C., Racot, M. \& BEAAICH, J. P. (1973). 'pH-stat' titration method for the determination of the thermodynamic quantities associated with the formation of the ATP-magnesium complex. Biochim. biophys. Acta 305, 1-10.

Sillan, L. G. \& Martell, A. E. (1964). Stability Constants of Metal-Ion Complexes, pp. 652-97. The Chemical Society, London.

Takahashi, K., Mori, T., Nakamura, H. \& Tonomura, Y. (1965). ATP-induced contraction of sarcomeres. F. Biochem. (Tokyo) 57, 637-49.

\section{EXPLANATION OF PLATE}

Fig. IOA, B. Multiple flash photomicrographs of Triton-extracted spermatozoa of $L y$ techinus reactivated in a solution containing $0.010 \mathrm{~mm} \mathrm{ATP}$ and $2.0 \mathrm{~mm}-\mathrm{MgSO}_{4}$. (A) Flash rate $\mathrm{I}_{4} \mathrm{~Hz}$. (B) Flash rate $2.8 \mathrm{~Hz}$.

Fig. roC-F. Multiple flash photomicrographs of Triton-extracted spermatozoa reactivated in solutions containing $0.24 \mathrm{mM}$ ATP and $0.70 \mathrm{mM}-\mathrm{MgSO}$. In Fig. Io F the sperm head is attached to the microscope slide. (C) Flash rate $26 \mathrm{~Hz}$. (D, E, F) Flash rate $16 \mathrm{~Hz}$; viscosity increased to about I I $\mathrm{cP}$ by addition of $0.3 \%$ methylcellulose.

Fig. IoG-I. Multiple flash photomicrographs of Triton-extracted spermatozoa reactivated in solutions containing $0.024 \mathrm{mM}$ ATP and $0.52 \mathrm{mM} \mathrm{MgSO}$. Flash rate $2.4 \mathrm{~Hz}$. For Fig. $\mathrm{H}$ the viscosity was increased by addition of $0.3 \%$ methyl-cellulose (I I cP) and for Fig. I the viscosity was increased by addition of $0.6 \%$ methylcellulose $(46 \mathrm{cP})$. 
